\title{
Rehabilitación integral en sextante anterior superior
}

\section{Comprehensive rehabilitation of the upper anterior sextant}

DOI: $10.46932 /$ sfjdv2n2-063

Received in: March 1st, 2021

Accepted in: May 30th, 2021

\section{Dra. Saralyn López y Taylor}

Universidad de Guadalajara Centro Universitario de Ciencias de la Salud, Departamento de Clínicas Odontológicas Integrales. México

Dra. Rosa Patricia Gómez Cobos

Universidad de Guadalajara Centro Universitario de Ciencias de la Salud, Departamento de Clínicas Odontológicas Integrales. México

Dra. Luz Elena Nápoles Salas

Universidad de Guadalajara Centro Universitario de Ciencias de la Salud, Departamento de Clínicas Odontológicas Integrales. México

E-mail: luznapoles06@hotmail.com

Dra. Belinda Pérez Santana

Universidad de Guadalajara Centro Universitario de Ciencias de la Salud, Departamento de Clínicas Odontológicas Integrales. México

\section{Dra. Sabrina del Rosario Pérez Santana}

Universidad de Guadalajara Centro Universitario de Ciencias de la Salud, Departamento de Clínicas Odontológicas Integrales. México

\section{Dra. Ana Bertha Olmedo Sanchez}

Universidad de Guadalajara Centro Universitario de Ciencias de la Salud, Departamento de Clínicas Odontológicas Integrales. México

\section{Dra. Olga Rocío Manzo Palomera}

Universidad de Guadalajara Centro Universitario de Ciencias de la Salud, Departamento de Clínicas Odontológicas Integrales. México

\section{RESUMEN}

La interrelación de las diversas especialidades de la odontología para la resolución de los tratamientos realizados en los pacientes que llegan a la consulta odontológica es hoy en día fundamental, ya que la visión sesgada de algún área nos puede privar el brindar una mejor posibilidad de tratamiento. Trabajar con especialistas en diferentes áreas conlleva a orientar adecuadamente los tratamientos y a optimizar resultados. En el presente caso se detalla la rehabilitación integral de un paciente con la participación de las especialidades endodoncia, periodoncia, rehabilitación oral, operatoria dental. El tratamiento multidisciplinario en odontología lleva a optimizar los resultados clínicos brindando una visión más amplia de las posibilidades de tratamiento para los pacientes.

Palabra Clave: Tratamiento multidisciplinario, rehabilitación Oral. 


\begin{abstract}
The interrelation of the different specialties of dentistry for the resolution of the treatments performed in patients who come to the dental office is fundamental nowadays, since the biased vision of any area can deprive us of providing a better possibility of treatment. Working with specialists in different areas leads to an adequate orientation of the treatments and to optimize results. In the present case the integral rehabilitation of a patient is detailed with the participation of the specialties endodontics, periodontics, oral rehabilitation and dental surgery. Multidisciplinary treatment in dentistry leads to optimize clinical results providing a broader vision of treatment possibilities for patients.
\end{abstract}

Keyword: Multidisciplinary treatment, oral rehabilitation.

\title{
1 INTRODUCCIÓN
}

La rehabilitación oral es una especialidad dentro de la odontología que ejerce en forma integral las áreas de prótesis fija, prótesis removible, operatoria, oclusión e implantologia. Para realizar una eficaz rehabilitación se necesita un diagnóstico y plan de tratamiento, en aquellos casos cuya complejidad es alta y son imprescindibles. De igual forma esta especialidad se relaciona horizontalmente, con las áreas de periodoncia, endodoncia, exodoncia y ortodoncia. La finalidad de esta área es cumplir con un equilibrio básico de función, integridad biológica y por supuesto la estética del paciente (1).

\section{EXPOSICIÓN DEL CASO}

Paciente masculino de 52 años de edad, acude a las Clínicas Odontológicas Integrales con motivo de consulta "Quiero que me dejen guapo". (figura. -1)

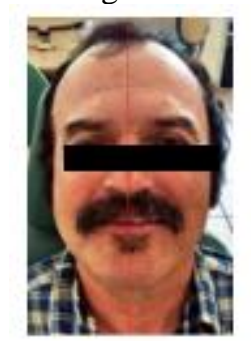

Su diagnóstico: Gingivitis asociada a placa, filtración en obturaciones metálicas y desgastes incisales en segmento anterior superior. Sé efectuó el encerado de diagnóstico, donde Vellini-Ferreira nos dice que nos servirá como una guía en nuestra rehabilitación final (2). (figura. -2)

Figura 2 


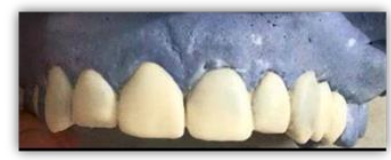

Se obtuvo una llave para los provisionales, se inició con el detartraje, y el tratamiento endodóntico de los cuatros órganos dentarios anteriores superiores (Figura 3 y 4).

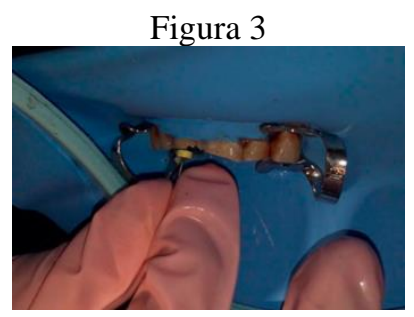

Figura 4

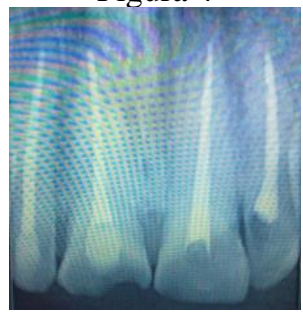

Para proseguir con la colocación de los postes metálicos prefabricados y la reconstrucción de los muñones (figura. -5 y 6 ).

Figura 5

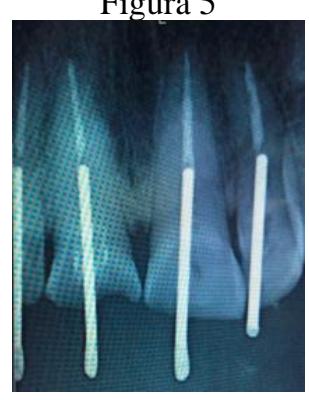

Figura 6

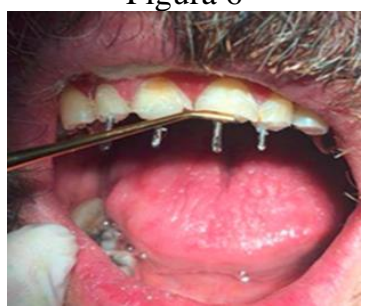

Se cementaron los postes metálicos prefabricados, se reconstruyeron los muñones para la preparación de los mismos (Figura. -7). 
Figura 7

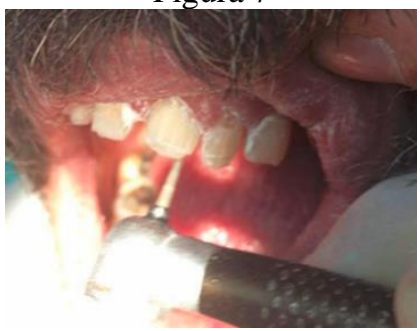

Se realizó la toma de impresión final, con polivinilsiloxano, y se obtuvo el modelo de trabajo. Se cementaron los provisionales, se mandó a laboratorio para obtener las cofias metálicas, en la prueba de metal lo importante es observar el sellado cervical de las cofias y los espacios que debe de existir para la colocación de la porcelana. Una vez que se revisó, se tomó color y se mandó al laboratorio para la aplicación de la porcelana a prueba de biscocho. Se hizo la prueba de biscocho y glaseado para su cementación final (Figura 8 y 9).

Figura 8

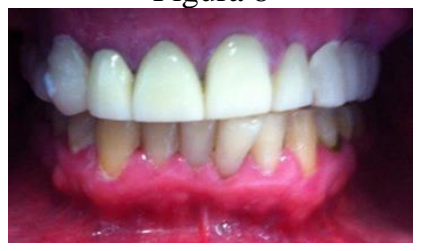

\section{OBJETIVO}

Restaurar los órganos dentarios afectados y dañados por medio de un tratamiento multidisciplinar.

\section{RESULTADOS}

La rehabilitación multidisciplinaria, nos arrojó un éxito, regresándole al paciente la función estética, fonética y el soporte labial, ayudándole a recobrar su autoestima.

\section{CONCLUSIÓN}

La complejidad de una rehabilitación completa se puede realizar siempre y cuando tengamos un buen diagnóstico y plan de tratamiento, según Jeffrey P. Okeson (5). Regresando soporte labial, estética y función, ya que con el desgaste de los bordes incisales se tiene una limitación para el corte de los alimentos (3) (4). 


\section{BIBLIOGRAFÍA}

1.Vellini-Ferreira, F. (2002). Ortodoncia, Diagnóstico y Planificación clínica. Ed. Artes Médicas Ltda. Sao Paulo- Brasil. Bermúdez J, Domínguez S, Suarez C, Jané L, RoigM.

2. BermudezJ, Domínguez S, Suarez C, Jane L, Roig M, Encerado de diagnóstico para el sector anterior. Revista de Especialidades Odontológicas. Volumen 1 num. 1-2, Epub, junio 2012.

3. Front Cover. Julio Barrancos Mooney, Patricio J. Barrancos. Operatoria dental: integración clínica Ed. Médica Panamericana, 2008 - 1345 pages

4. Leonardo. Endodoncia: Conceptos Biológicos y Recursos Tecnológicos ,2009

5. Jefferey P. Okeson, Tratamiento de Oclusión y afecciones temporomandibulares 8va edicion 2019. 